\title{
Model for Cumulative Solar Heavy Ion Energy and Linear Energy Transfer Spectra
}

\author{
M. A. Xapsos, Senior Member, IEEE, C. Stauffer, T. Jordan, J. L. Barth, Senior Member, IEEE, and R. A. Mewaldt
}

\begin{abstract}
A probabilistic model of cumulative solar heavy ion energy and LET spectra is developed for spacecraft design applications. Spectra are given as a function of confidence level, mission time period during solar maximum and shielding thickness. It is shown that long-term solar heavy ion fluxes exceed galactic cosmic ray fluxes during solar maximum for shielding levels of interest. Cumulative solar heavy ion fluences should therefore be accounted for in single event effects rate calculations and in the planning of space missions.
\end{abstract}

Index Terms-Cumulative fluence, solar heavy ion, solar particle event.

\section{INTRODUCTION}

$\mathbf{T}$ HE effects that solar particle events have on microelectronics, photonics, detectors and solar arrays are important to account for during the design phase of spacecraft. This is particularly true for missions away from the protection of the Earth's magnetic field such as geosynchronous, polar and interplanetary missions. Astronaut exposure is also a serious concern for new exploration initiatives.

One of the important considerations for spacecraft designers is the accumulation of solar proton fluences over the duration of a mission. Probabilistic models have most often been used for this purpose so that risk-cost-performance tradeoffs can be evaluated. There are a number of solar proton models for engineering design applications and comparisons of some of the commonly used models are shown in Fig. 1 for the $90 \%$ confidence level and 2 year period during solar maximum [1].

The Jet Propulsion Laboratory 1991 (JPL91) Model [2], Emission of Solar Protons (ESP) Model [3], and Prediction of Solar particle Yields for CHaracterizing Integrated Circuits (PSYCHIC) Model [4] all agree reasonably well in their overlapping energy ranges, while the King Model [5] is somewhat different because it is based on the energy spectrum of the well-known August 1972 event.

One of the advantages of the PSYCHIC Model that is apparent from this figure is its considerably higher energy range, which extends to over $300 \mathrm{MeV}$. This is significant for proton-

Manuscript received July 19, 2007; revised September 6, 2007. This work was supported in part by the NASA Goddard Space Flight Center Core Capability Development Program and by the NASA Constellation Program.

M. A. Xapsos and J. L. Barth are with the NASA Goddard Space Flight Center, Greenbelt, MD 20771 USA (e-mail: Michael.A.Xapsos@nasa.gov).

C. Stauffer is with MEI Technology Inc., Seabrook, MD 20706 USA.

T. Jordan is with EMP Consultants, Gaithersburg, MD 20885 USA.

R. A. Mewaldt is with the California Institute of Technology, Pasadena, CA 91125 USA.

Digital Object Identifier 10.1109/TNS.2007.910850

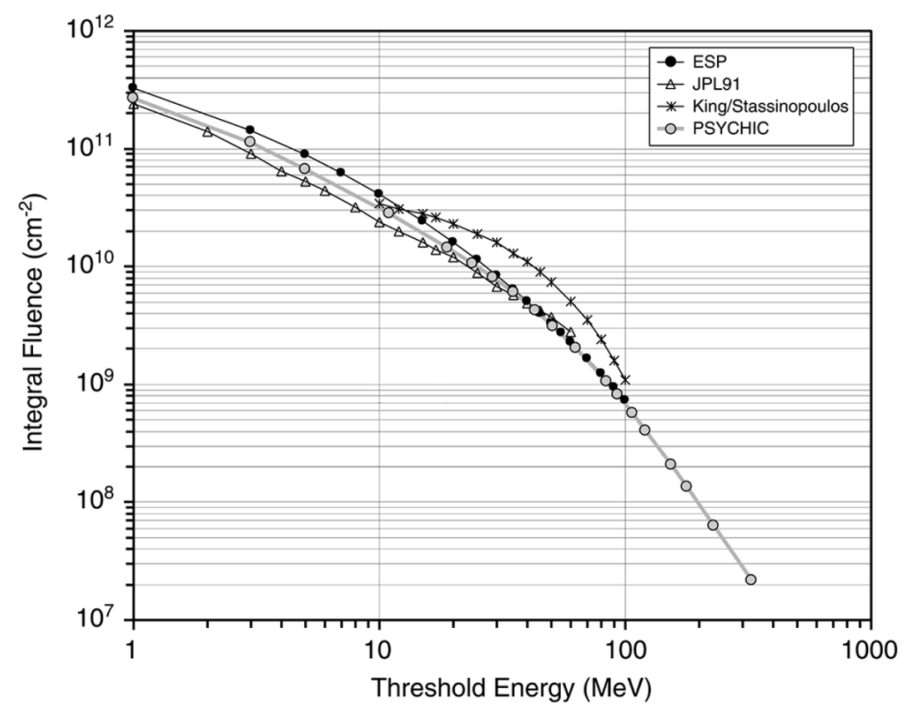

Fig. 1. Comparison of solar proton cumulative fluence models for a 2-year mission during solar maximum at the $90 \%$ confidence level [1].

induced single event effects (SEE), total ionizing dose and displacement damage effects in heavily shielded regions of spacecraft and astronaut exposure. The data base is rather extensive, incorporating measurements made on the Interplanetary Monitoring Platform (IMP) and Geostationary Operational Environmental Satellites (GOES) series of spacecraft spanning a time period from 1966 to 2001. Probability distributions for solar proton event magnitudes and cumulative fluences are derived from Maximum Entropy Theory [1], [3]. This provides a mathematical procedure for selecting a probability distribution when the data are limited. Use of the cumulative lognormal function to model cumulative fluences has been validated by both simulation and direct analysis of the data [3].

Solar heavy ion models are not nearly as advanced as solar proton models mainly because of the relative lack of heavy ion data. The significance of the contribution of solar heavy ions to radiation effects has been difficult to assess because of the lack of a detailed quantitative model of cumulative exposure. Solar heavy ions are generally believed to be a concern for SEE considerations, and the possibility has been pointed out that alpha particles may contribute significantly to the degradation of photovoltaics [6]. However, the contribution to effects such as total ionizing dose and displacement damage is often disregarded in spacecraft design. Solar heavy ions may also be a concern for manned missions because of the effectiveness of highly charged ions in producing biological damage.

In this work, the PSYCHIC model of solar protons is extended to include nearly all naturally occurring elements across 
the Periodic Table. The result is a detailed probabilistic model of cumulative solar heavy ion fluences for both incident ion energy and linear energy transfer (LET) spectra during the solar maximum time period. Spectra are presented for different shielding thicknesses appropriate for modern spacecraft. The main application in mind is for SEE rate predictions and design specifications. Since the galactic cosmic ray (GCR) environment is commonly used for long-term SEE assessments the new solar heavy ion results are benchmarked by comparison with GCR fluxes during solar maximum. In addition, the long-term dose deposited by solar heavy ions in silicon behind aluminum shielding is evaluated.

\section{DATA AND METHODS}

\section{A. Alpha Particles}

Alpha particle data were analyzed in a manner similar to how the proton data were analyzed for the PSYCHIC model [4], aimed at combining the best features of the IMP and GOES data sets. The Goddard Medium Energy (GME) data from the IMP-8 satellite were used to obtain the alpha particle fluxes during solar particle events in the time period from 1973 to 2001. This satellite is well positioned to measure interplanetary fluxes in an approximately circular orbit at 35 Earth radii. There were 26 energy bins of alpha particle differential fluxes used that spanned the energy range of 1.1 to $250 \mathrm{MeV}$ per nucleon. The main problem with this instrument is that the detectors saturate during very large fluxes. The GOES data have thus been used to fill in the gaps in the GME data when the GME instrument is saturated. The GOES data that were used were taken by the Space Environment Monitor (SEM) instrumentation during the time period from 1986 to 2001. These data are available in 6 energy bins ranging from energies of 1 to $125 \mathrm{MeV}$ per nucleon. The resulting alpha particle energy spectra derived from the combined instrumentation have been summed up for the solar maximum periods between 1973 and 2001. This amounts to about 18 years of data. The resulting summed energy spectrum was compared to the analogous solar proton energy spectrum over the same time period and from the same instrumentation. This gives the scaling relation of the 2 spectra relative to each other in a long-term average sense. The result is used to calculate the alpha particle energy spectrum relative to the proton energy spectrum. The proton spectrum is obtained from the PSYCHIC model using a confidence level and mission time period as input.

\section{B. Major Heavy Elements}

The previous section described how the alpha particle energy spectrum is calculated once the proton energy spectrum is known. This section describes how the model energy spectra of the major heavy elements with $\mathrm{Z}>2$ are calculated once the alpha particle energy spectrum is known. The data used are measurements from the Solar Isotope Spectrometer (SIS) instrument onboard the Advanced Composition Explorer (ACE) spacecraft. This modern instrumentation provides excellent mass resolution during the high flux rates that occur during solar particle events. It has been operational since 1997 and the data used in the current model cover the most recent 7-year solar maximum time period, which is taken to be calendar years 1998 through 2004, inclusive. The available energy spectra measured by this instrumentation are for alpha particles, $\mathrm{C}, \mathrm{N}, \mathrm{O}, \mathrm{Ne}, \mathrm{Mg}, \mathrm{Si}, \mathrm{S}$ and Fe. The spectra due to solar particle events were summed for each element over the 7-year period. This result then gives the long-term average energy spectra of the 8 elements $\mathrm{C}$ through $\mathrm{Fe}$ listed above, relative to alpha particles. Thus, the current model is based on long-term measured energy spectra of all the major elements.

\section{Minor Heavy Elements}

Examination of the most current known elemental abundances in the solar photosphere given by Grevesse and Sauval shows that the 10 elements discussed above ranging from protons $(\mathrm{Z}=1)$ through $\mathrm{Fe}(\mathrm{Z}=26)$ are indeed the dominant elements in the solar particle environment [7]. Estimates of the energy spectra of other naturally occurring elements in the Periodic Table are included in our solar heavy ion model, even though in many cases the elemental abundances are orders of magnitude less than the dominant elements. Reference [7] gives no data for the 9 elements with atomic numbers $\mathrm{Z}=43,61,84-89$ and 91 , presumably because there are no reliable measurements. The relative abundances of elements surrounding these missing data are 5 to 7 orders of magnitude less than that of $\mathrm{Fe}(Z=26)$. These 9 elements have therefore not been included in our model. Their omission is expected to have a negligible effect.

The following procedure is used for estimating energy spectra for minor heavy elements with $Z>2$. The energy spectrum of the minor element is taken to be a fraction of the energy spectrum of the closest major element in the Periodic Table. For 11 elements $(\mathrm{Z}=11,13,15,17-20,22,24,28,30)$ this fraction was determined from measurements on the International SunEarth Explorer-3 (ISEE-3) spacecraft, using the 5-12 MeV per nucleon energy range for 49 large solar particle events that occurred over a 14-year period [8]. The fractions for the remaining minor elemental energy spectra were determined from an abundance model. This started from current knowledge of solar photospheric abundances given in [7]. However, it is a well-known empirical observation that the elemental abundances seen in solar particle events are enhanced over the photospheric abundance by about a factor of 3 if the first ionization potential of the element is less than about $10 \mathrm{eV}$ [9]. This is the so-called First Ionization Potential (FIP) effect. This factor of 3 correction is made in our abundance model energy spectra.

In this manner our model fills in the naturally occurring minor elements in the Periodic Table. If examined in terms of an LET spectrum, the most noticeable contribution from these elements is seen for LET values greater than about $29 \mathrm{MeV}-\mathrm{cm}^{2} / \mathrm{mg}$. This comes from elements heavier than Fe. Since it is possible these trans-iron elements can impact SEEs, our model results were checked against measurements made with the Low-Energy Matrix Telescope (LEMT) on the WIND spacecraft. This spacecraft is in a halo orbit around the earth-sun L1 Lagrange point. The available data are in the 3.3 to $10 \mathrm{MeV}$ per nucleon energy range over a period of nearly 6 years during solar maximum [10]. Due to the low counting rates of these heavy elements only upper and lower limits for $34 \leq \mathrm{Z} \leq 40$, for $50 \leq \mathrm{Z} \leq 56$, and an upper limit for the $76 \leq \mathrm{Z} \leq 82$ group have been analyzed from the 


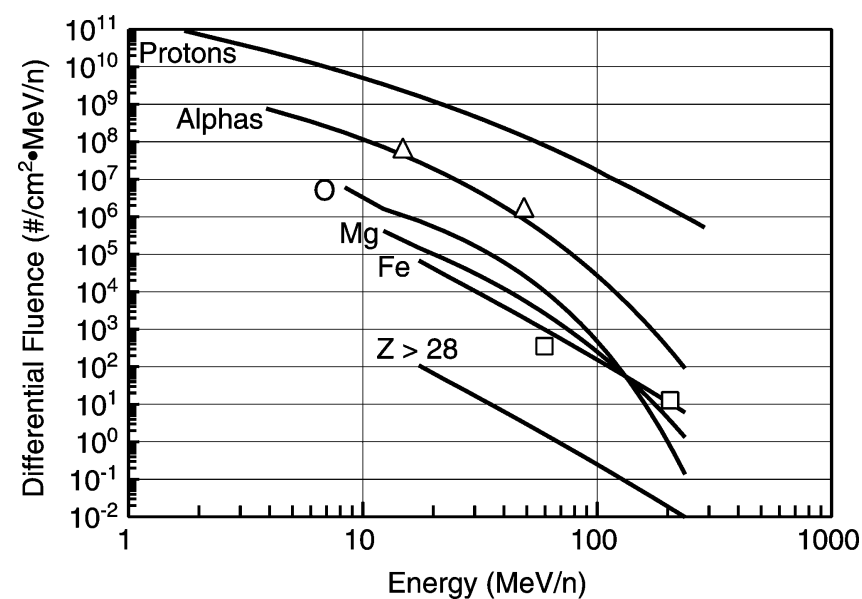

Fig. 2. Differential fluence-energy spectra for protons, alpha particles, oxygen, magnesium, iron and summed spectra for $Z>28$ elements for a 2-year mission during solar maximum at the $90 \%$ confidence level. Triangles and squares are results for alpha particles and iron, respectively, obtained from [6].

LEMT data. Our model is consistent with these limits, giving us confidence that the model energy spectra for minor elements are reasonable.

\section{RESULTS}

Fig. 2 shows results for differential energy spectra for 5 of the major elements and a summed spectrum for all elements with $\mathrm{Z}>28$. The example calculation was done for a 2-year period during solar maximum at the $90 \%$ confidence level. This is interpreted to mean there is a $90 \%$ chance the fluence levels will not be exceeded during a 2-year mission that occurs during solar maximum. Since the shape of the energy spectra vary significantly with $\mathrm{Z}$, it is apparent that a realistic model would have to incorporate spectra of the major elements that are based on actual space data. That is the case here. The energy spectra in the figure extend to high enough energies that the high-energy fluxes are small compared to the background galactic cosmic ray (GCR) fluxes at the same energy [1]. On the low energy side, the cut-off energy point is based on shielding considerations. We have determined that it takes at most 10 mils of aluminum shielding to stop all the lowest energy particles in the model energy spectra. It is therefore recommended that the model be used for situations where the shielding is at least an equivalent 10 mils of aluminum. Although this is not a limitation for most applications we are currently investigating relaxing this constraint by extending the energy spectra to lower energies based on the results of Mewaldt, et al.. [11].

Also shown by the points in Fig. 2 are results for cumulative heavy ion exposure given by Tylka based on IMP-8 data taken with the University of Chicago cosmic ray telescope [6]. The triangles are for alpha particles in the 11-20 and 25-95 MeV per nucleon range, while the squares are for $\mathrm{Fe}$ in the $45-79$ and $97-432 \mathrm{MeV}$ per nucleon range at the same confidence level and mission duration as our results. The points are plotted at the logarithmic average of each energy range. Despite the limitations of the energy spectra in the Tylka study, the results are nonetheless useful for comparison purposes. We find there is fair agreement between the two modeling efforts although some differences do

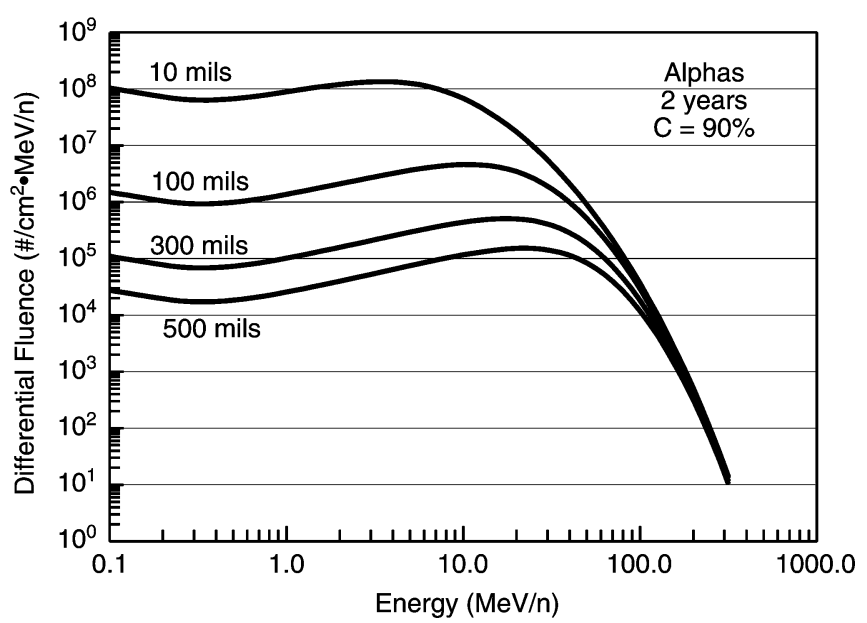

Fig. 3. Differential fluence-energy spectra for alpha particles accumulated over a 2 -year period at the $90 \%$ confidence level. Results are for shielding by 10,100 , 300 and 500 mils of aluminum in a solid sphere geometry.

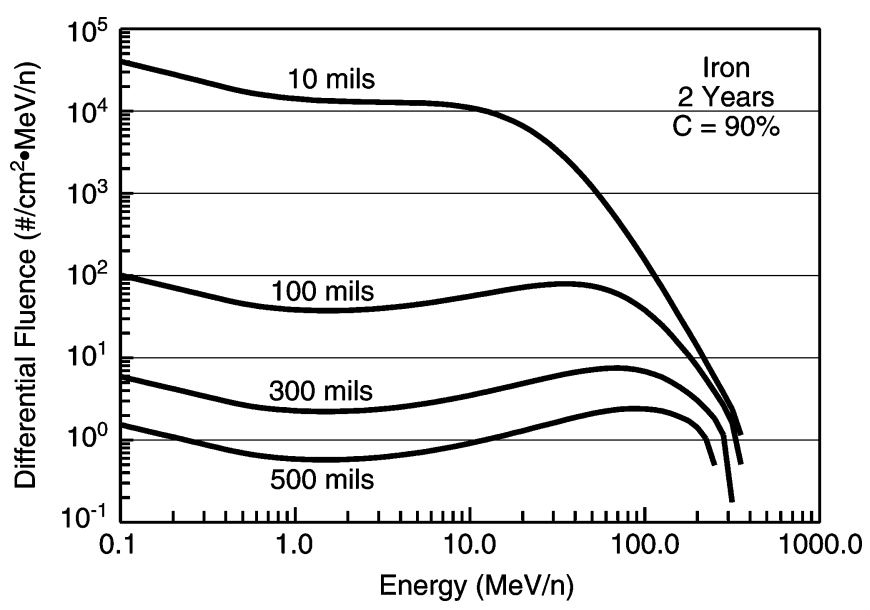

Fig. 4. Differential fluence-energy spectra for iron ions accumulated over a 2 -year period at the $90 \%$ confidence level. Results are for shielding by 10,100 , 300 and 500 mils of aluminum in a solid sphere geometry.

exist such as the factor of approximately 3 at the low energy iron point from [6].

Figs. 3 and 4 show the effect of shielding on the differential energy spectra of alpha particles and iron from the lightly shielded out to the heavily shielded cases of 10 and 500 mils aluminum. The geometry assumed is that of a solid sphere. The shielding calculations were done with the NOVICE code [12] and cover a range of thickness that is often typical for modern spacecraft. Again this is for a 2-year mission at the $90 \%$ confidence level. Knowing the elemental energy spectra can be important for some SEE applications in modern devices. The traditional LET metric may not be useful because of the highly scaled nature of devices, the complexity of the geometry, or the increased use of metal over-layers. In addition, it does not typically account for nuclear reaction products that can cause SEE.

The LET metric is nonetheless still a useful and convenient parameterization. It transforms the energy spectra of all elements of concern to a single curve of fluence vs. LET. Fig. 5 shows the effect that shielding has on LET spectra that include all the elements in our model. Note that the high LET values are 


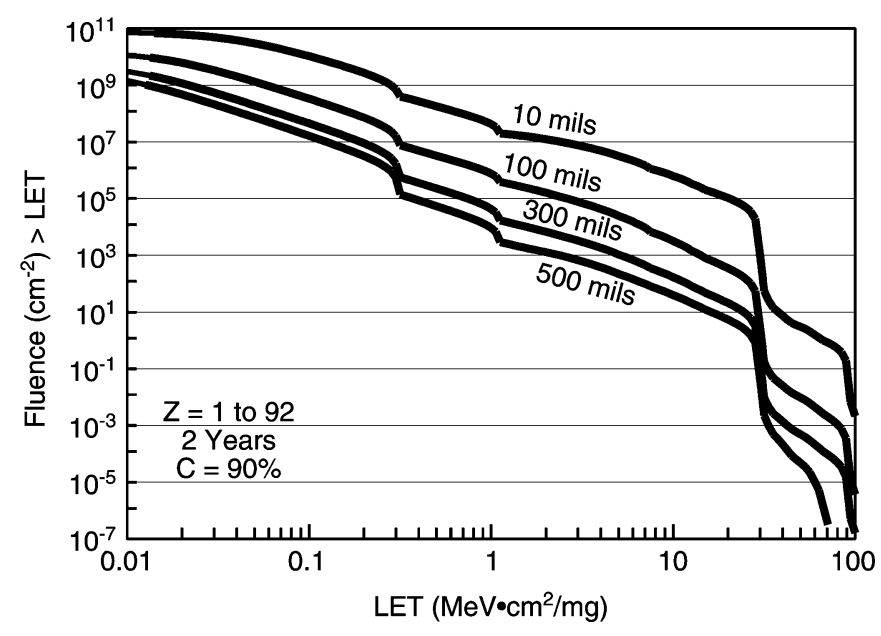

Fig. 5. Integral LET spectra for cumulative solar particle events over a 2-year solar maximum period at the $90 \%$ confidence level. Results include elements from atomic number $Z=1$ to 92 . Curves are for shielding thickness of 10 , 100,300 and 500 mils of aluminum in a solid sphere geometry.

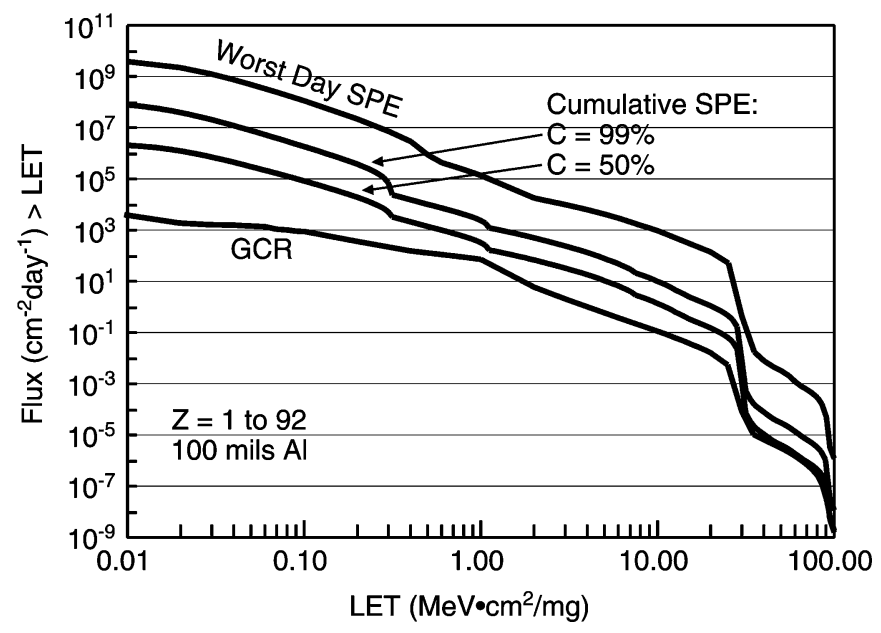

Fig. 6. Integral LET spectra obtained from CREME96 [13] for the "worst day" solar particle event (top curve) and GCR during solar maximum (bottom curve) compared to cumulative solar particle event spectra at the $50 \%$ and $99 \%$ confidence levels. All results are for 100 mils of aluminum shielding.

reduced more quickly with added shielding than the low LET values. Thus, higher LET particles in solar particle events are more easily shielded against than the lower LET particles.

Fig. 6 benchmarks our new model results for LET spectra against results from the widely used CREME96 program suite for the solar maximum time period [13]. GCR fluxes are often used alone for long-term SEE rate assessment. Thus, the significance of the solar particle fluxes in this context lies with a direct comparison to the GCR fluxes at the same shielding thickness, taken as 100 mils of aluminum. This is shown in Fig. 6 for two situations. First we use the $50 \%$ confidence level to represent the long-term average solar particle event (SPE) flux. This would be appropriate for evaluating an average SEE rate due to solar particles. Secondly, we show the $99 \%$ confidence level, appropriate for a conservative design estimate of the SEE rate due to solar particles. The long-term SPE fluxes exceed the GCR flux for both cases, indicating that SPE heavy ion fluxes are an important consideration during solar maximum at a shielding

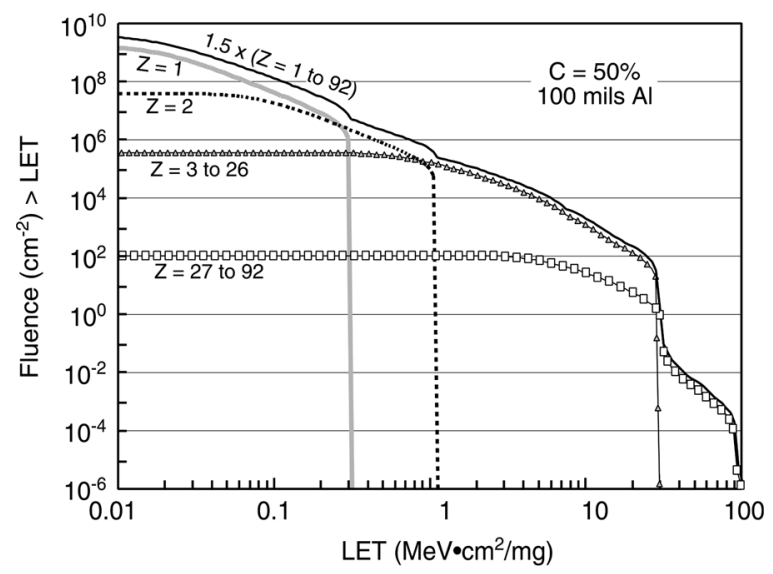

Fig. 7. Integral LET spectra for cumulative solar particle events for a 2-year mission during solar maximum at the $50 \%$ confidence level. The spectra illustrate the contributions of $Z=1$ (gray line), $Z=2$ (dashed line), $Z=3$ to 26 (triangles), and $Z=27$ to 92 (squares) ions to the total spectrum (solid black line). The total has been multiplied by a factor of 1.5 for clarity.

depth of 100 mils of aluminum. The ratio of the $50 \%$ confidence level SPE flux to the GCR flux shown in Fig. 6 is about 16 at an LET of $3 \mathrm{MeV}-\mathrm{cm}^{2} / \mathrm{mg}$. This ratio decreases to about 12 at an LET of 10, and about 9.4 at an LET of 20. Solar heavy ions are more easily shielded against than GCR so as the shielding thickness increases the differences shown in Fig. 6 will decrease, especially at the high LET values. Nonetheless, even for more heavily shielded regions in spacecraft that may be equivalent to a few hundred mils of aluminum, the SPE fluxes are comparable to the GCR fluxes.

Also shown in Fig. 6 for comparison purposes is the "worst day" solar particle event model result from CREME96. This is based on the highest flux observed during the well-known October 1989 event averaged over a 1-day period as determined from GOES-6 and -7 instrumentation. The heavy ion fluxes are determined from measurements of the University of Chicago Cosmic Ray Telescope and an abundance model. The "worst day" results are the highest fluxes shown in the figure, as expected. Analysis of the cumulative SPE results along with GCR fluxes and worst-case results such as those shown in the figure allows a more thorough assessment of SEE effects.

LET spectra are typically composed of a large number of elements ranging across the Periodic Table. Examining the spectra in terms of elemental components gives further insight into the model as well as some key radiation effects metrics. This is shown in Fig. 7 for the $50 \%$ confidence level, which is representative of long-term average SPE fluences. The total fluence for all elements shown in the figure is multiplied by a factor of 1.5 for clarity. This is composed of a proton curve $(Z=1)$ and an alpha particle curve $(Z=2)$. These are high fluence, low LET components where the direct ionization contribution drops sharply to zero at the so-called "Bragg Peak" or maximum LET. The contribution from $Z=3$ to 26 particles extends out to an LET of about $29 \mathrm{MeV}-\mathrm{cm}^{2} / \mathrm{mg}$. The fluence contribution from this group is dominated by the 8 major elements whose energy spectra were measured by the ACE spacecraft instrumentation. For many SEE applications this group of elements has the greatest impact on SEE rates. Finally, the 
trans-iron group ( $Z=27$ to 92 ) extends out to an LET of over $100 \mathrm{MeV}-\mathrm{cm}^{2} / \mathrm{mg}$. This is composed mainly of elements in our abundance model.

Since solar protons are often considered to be the dominant SPE contribution to total ionizing dose effects, it is of interest to evaluate this assumption quantitatively. We have calculated the long-term SPE dose deposited in silicon behind 100 mils of $\mathrm{Al}$ shielding. The $50 \%$ confidence level is again chosen to represent average SPE energy spectra. We find that about $90 \%$ of the dose is deposited by protons, $9 \%$ by alpha particles, and $1 \%$ by heavier ions.

\section{SUMmary AND CONCLUSIONS}

In this work we have developed a new statistical model of cumulative solar heavy ion fluences during the solar maximum time period. It is based on well-established probabilistic methods, analysis of long-term satellite measurements of solar particle events and current knowledge of the chemical composition and processes in the solar photosphere. This adds to the available space radiation environment design tools and is especially important for SEE considerations. It allows calculations of both solar particle energy spectra and LET spectra to be obtained for different mission time periods, levels of confidence and shielding.

There are significant conclusions that can be drawn by comparison of the new model results with GCR spectra. First and most importantly, the long-term SPE fluxes exceed GCR fluxes during solar maximum at shielding levels often considered for spacecraft design, i.e., 100 mils of aluminum. Solar heavy ions are more easily shielded against than GCR, especially in the high LET region so shielding analysis is also significant. However, even in the more heavily shielded regions of spacecraft the SPE fluxes can be comparable to the GCR fluxes. Therefore, long-term SPE fluxes should be considered for SEE rate calculations.
In order to make this model available, we are working toward distributing it as a stand-alone code with a Windows ${ }^{\text {TM }}$ interface. We also plan to make it available to widely used program suites such as SPENVIS and CREME.

\section{ACKNOWLEDGMENT}

M. A. Xapsos thanks Don Reames of the NASA Goddard Space Flight Center for helpful discussion about solar heavy ion abundances.

\section{REFERENCES}

[1] M. A. Xapsos, "Modeling the Space Radiation Environment," presented at the IEEE Nuclear and Space Radiation Effects Conf. (Short Course), Ponte Vedra Beach, FL, Jul. 2006.

[2] J. Feynman, G. Spitale, J. Wang, and S. Gabriel, "Interplanetary fluence model: JPL 1991," J. Geophys. Res., vol. 98, pp. 13281-13294, 1993.

[3] M. A. Xapsos, G. P. Summers, J. L. Barth, E. G. Stassinopoulos, and E. A. Burke, "Probability model for cumulative solar proton event fluences," IEEE Trans. Nucl. Sci., vol. 47, no. 3, pp. 486-490, Jun. 2000.

[4] M. A. Xapsos, C. Stauffer, G. B. Gee, J. L. Barth, E. G. Stassinopoulos, and R. E. McGuire, "Model for solar proton risk assessment," IEEE Trans. Nucl. Sci., vol. 51, no. 6, pp. 3394-3398, Dec. 2004.

[5] J. H. King, "Solar proton fluences for 1977-1983 space missions," $J$. Spacecraft, vol. 11, pp. 401-408, 1974.

[6] A. J. Tylka, W. F. Dietrich, and P. R. Boberg, "Probability distributions of high-energy solar-heavy-ion fluxes from IMP-8: 1973-1996," IEEE Trans. Nucl. Sci., vol. 44, no. 6, pp. 2140-2149, Dec. 1997.

[7] N. Grevesse and A. J. Sauval, "Standard solar composition," Space Sci. Rev., vol. 85, pp. 161-174, 1998.

[8] D. V. Reames, "Solar energetic particles: Sampling coronal abundances," Space Sci. Rev., vol. 85, pp. 327-340, 1998.

[9] D. V. Reames, "Particle acceleration at the sun and in the heliosphere," Space Sci. Rev., vol. 90, pp. 413-491, 1999.

[10] D. V. Reames and C. K. Ng, "Heavy-element abundances in solar energetic particle events," Astrophys. J., vol. 610, pp. 510-522, July 2004.

[11] R. A. Mewaldt, C. M. S. Cohen, G. M. Mason, D. K. Haggerty, and M I. Desai, "Long-Term Fluences of Solar Energetic Particles from $\mathrm{H}$ to Fe," Space Sci. Rev., submitted for publication.

[12] NOVICE Software Documentation. Gaithersburg, MD, 2006, E.M.P. Consultants.

[13] A. J. Tylka et al., "CREME96: A revision of the cosmic ray effects on microelectronics code," IEEE Trans. Nucl. Sci., vol. 44, no. 6, pp. 2150-2160, Dec. 1997. 